\title{
Efficacy of a Mosaic Long-Lasting Insecticide Net, PermaNet 3.0, against Wild Populations of Culex quinquefasciatus in Experimental Huts in Togo
}

\author{
Dominic B. Dery, ${ }^{1}$ Guillaume K. Ketoh, ${ }^{2}$ Joseph Chabi, ${ }^{3}$ George Apetogbo, ${ }^{2}$ \\ Isabelle A. Glitho, ${ }^{2}$ Thierry Baldet, ${ }^{3}$ and Jean-Marc Hougard ${ }^{3,4}$ \\ ${ }^{1}$ Kintampo Health Research Centre, Ghana Health Service, Ministry of Health, P.O. Box 200, Kintampo, Ghana \\ ${ }^{2}$ Laboratoire d'Entomologie Appliquée (LEA), Faculté des Sciences, Université de Lome, P.O. Box 1515, Lomé, Togo \\ ${ }^{3}$ Centre de Recherche Entomologique de Cotonou (CREC), Institut de Recherche Pour le Développement (IRD), 01 P.O. Box 4414 RP, \\ Cotonou, Benin \\ ${ }^{4}$ Anopheles Biology and Control (ABC) Network, Institut de Recherche Pour le Développement (IRD), P.O. Box 1386, Dakar, Senegal
}

Correspondence should be addressed to Dominic B. Dery; bonereme@gmail.com

Received 1 October 2012; Accepted 30 October 2012

Academic Editors: K. Couper, M. A. De Souza, H. Hisaeda, and D.-D. Ji

Copyright (C) 2013 Dominic B. Dery et al. This is an open access article distributed under the Creative Commons Attribution License, which permits unrestricted use, distribution, and reproduction in any medium, provided the original work is properly cited.

PermaNet 3.0 was evaluated against Culex quinquefasciatus in experimental huts in Lomé. Endpoints were deterrence, exophily, blood feeding inhibition, and mortality. Insecticide susceptibility of Culex quinquefasciatus was assessed with permethrin (1\%), DDT (4\%), bendiocarb $(0.1 \%)$, deltamethrin $(0.5 \%, 0.05 \%)$, carbosulfan $(0.4 \%)$, and chlorpyrifos methyl $(0.4 \%)$. Total of 1,223 Cx. quinquefasciatus females were collected. PermaNet 3.0 unwashed deterred $16.84 \%$ Culex mosquitoes. After 20 washes, it deterred 5.79\% mosquitoes compared to $6.84 \%$ deterrence by unwashed PermaNet 2.0. PermaNet 3.0 induced mosquitoes to exit huts 50.48\% and inhibited blood feeding $70.97 \%$ in unwashed state. After 20 washes, the net induced $42.91 \%$ mosquitoes to exit and inhibited $67.06 \%$ mosquitoes from blood feeding. PermaNet 3.0 gave $76 \%$ personal protection at zero wash and $69 \%$ protection after 20 washes. The net retained $7.1 \%$ insecticidal effect in the unwashed state and after 20 washes $6.5 \%$. Cx. quinquefasciatus was resistant to tested insecticides $(6 \%-50 \%$ mortality). PermaNet 3.0 is a good control tool against mosquitoes. However, Cx. quinquefasciatus is less affected by PermaNet 3.0. The evaluation depicts the success of vector control innovations using pyrethroids and nonpyrethroids in combination on nets. Additional studies with Culex species are recommended to know whether the difference in blood feeding is interspecific (difference in vector behaviour) and not due to intertrial variability.

\section{Background}

Insecticide treated nets are recommended by the World Health Organisation (WHO) for malaria control in malaria endemic countries [1-3] and for control of other diseases of medical importance [4]. They offer individual and community protection against malaria $[3,5]$, sometimes reducing morbidity by as much as $50 \%$ and global mortality by $20-30 \%$ [6]. New techniques for long-lasting insecticide treatment of nets provide solution for the need to regularly re-treat nets
[7]. For acceptability at community level, nets should as well offer protection to individuals against nuisance vectors such as Culex and Mansonia mosquito species [5, 8, 9].

Many infectious diseases in Africa are caused by Culex mosquitoes [10] such as West Nile Virus (WVN), Rift Valley Fever (RVF) and Bancroftian filariasis [4]. Most organized efforts to control populations of $C$. quinquefasciatus ( $C$. fatigans, C. pipiens fatigans, and C. pipiens quinquefasciatus) in Asia and elsewhere have been directed at the immature stages $[11,12]$. Most Culex species rest outdoors, except for 
C. quinquefasciatus, which is a domestic mosquito, over $50 \%$ resting on nonsprayable surfaces in houses, such as mosquito nets, clothes, hangings and furniture [13].

Directly and indirectly, the use of pesticides has resulted in selection of broad spectrum organophosphate and carbamate resistance against C. Quinquefasciatus [14], and pyrethroid resistance with the most commonly selected resistance mechanism observed as increased activity of Esta ${ }^{2}$ and Estb ${ }^{2}$ carboxylesterases; $\mathrm{A} 2$ and $\mathrm{B} 2$ esterases on an earlier classification $[15,16]$. One mechanism of resistance to pyrethroids is increased reduction in intrinsic insensitivity of the insect nervous system [17], and a second mechanism involves increased insecticide metabolism [18].

Currently, pyrethroid insecticides are the recommended compounds for the treatment of mosquito nets for malaria control [19], because they have low mammalian toxicity and fast acting properties against mosquitoes [20]. Resistance in many disease vectors to pyrethroids threatens this recommendation and jeopardises the success of spraying efforts and bed net impregnation [21]. Several strategies have been proposed for resistance management. Notably, to use a pyrethroid and a nonpyrethroid insecticide in combination on the same mosquito net, either separately or as a mixture [22]. A few studies have shown that exposure to the P450 monooxygenase inhibitor, piperonyl butoxide (PBO) caused a partial reversal of Permethrin resistance [23]. Piperonyl butoxide $(\mathrm{PBO})$ is a pesticide synergist. It does not have pesticidal properties. However, when added to insecticide mixtures, typically pyrethrin, pyrethroid, and carbamate insecticides, their potency is increased considerably. It is a potent cytochrome P450 inhibitor and this family of enzymes acts as the principal detoxification pathway for many pesticides. Inhibiting the detoxification pathway allows higher unmetabolised systemic concentrations of the active insecticide to remain within the target insect for a longer period.

Urbanization continues to create favourable grounds for breeding of nuisance mosquitoes such as Culex, with strong levels of pyrethroid resistance $[24,25]$. Developing alternative chemicals and/or vector control strategies using long-lasting insecticide nets (LLIN) to maintain an effective control of resistant mosquito populations has, therefore, become a priority. New methods and strategies are urgently needed [20], and regular resistance surveillance or monitoring research activities timely are welcomed.

Due to recent developments in bioactive fiber technology, other long-lasting insecticide-treated materials, such as curtains, tents, clothing, blankets, and plastic sheeting have been tested as potential alternatives to mosquito nets [21, 26]. However, they proved to be effective only in addition to mosquito nets or in limited epidemiological or cultural settings $[1,19]$.

Similar to a multicentre WHO commissioned phase II trial [27], this research evaluated the new technology net (PermaNet 3.0) in verandah-trap huts in Togo against freeflying wild Culex quinquefasciatus. Efficacy was expressed in terms of blood feeding inhibition, deterrence, induced exophily, and mortality. In parallel, susceptibility of Culex quinquefasciatus to four groups of insecticides (DDT, permethrin, carbosulfan, deltamethrin, bendiocarb, and chlorpyrifos methyl) was evaluated with and without a synergist (PBO).

\section{Methods}

2.1. Study Area. Togo lies $6.17^{\circ}$ north, $1.35^{\circ}$. It is bordered by Burkina Faso to the north, Benin to the east, and Ghana to the west. The study area, Akodésséwa district, is situated in the urban district in the eastern part of Lomé with vibrant economic activities. The area is characterized by scattered water bodies, inland water impoundments, and water run-offs from the Bè lagoon. Vegetable crop cultivation is predominant along the boundaries of the Lagoon at the periphery closer to the Atlantic sea. There are also scattered settlements along the boundaries of the lagoon and various activities such as cars, trucks, and stone washing bays designated at intervals primarily due to the available water source from the lagoon.

2.2. Specifications of Nets Used for Evaluation. PermaNet 3.0 is manufactured by Vestergaard Frandsen SA. All the nets in the trial were of the same size: $120 \mathrm{~cm} \times 190 \mathrm{~cm} \times 150 \mathrm{~cm}$. PermaNet 2.0 had specification as follows: deltamethrin $\left(25 \mathrm{mg} / \mathrm{m}^{2}\right)$ coated on polyethylene monofilament net fabric. PermaNet 3.0 has the following specification: $85 \mathrm{mg} / \mathrm{m}^{2}$ deltamethrin in net and $115 \mathrm{mg} / \mathrm{m}^{2}$ in $70 \mathrm{~cm}$ boarder (i.e., $2.8 \mathrm{~g} / \mathrm{kg}$ deltamethrin load in 75 denier net fabric). The roof of the net (upper part) had deltamethrin and piperonyl butoxide (PBO) incorporated into the monofilament (220 denier with $4 \mathrm{~g} / \mathrm{kg}$ deltamethrin; $15 \mathrm{~g} / \mathrm{kg}$ PBO).

2.3. Initial Treatment of Nets prior to Trial. For the 20 minimum WHO resistance to washing, nets were washed with "savon de Marseille" $2 \mathrm{~g} /$ litre (soap: $\mathrm{H}_{2} \mathrm{O}$ ) with approximately 2 degree hardness tap water. Washing was done in a total of 10 litres of water $\left(\mathrm{H}_{2} \mathrm{O}\right)$ for 3 minutes left for 4 minutes and washed again for 3 minutes using a wooden ladle (each wash agitation in different direction of rotation). They were rinsed twice in 10 litres of clean water and air dried in shade. Subsequent washes were repeated on each day for 20 days (20 times), 18th January-6th February, 2008. Before the start of the trial, nets were deliberately holed; six holes per net $2 \mathrm{~cm} \times 2 \mathrm{~cm}$. Two holes located on the longer sides and one hole each on the shorter sides.

\subsection{Efficacy and Effect of Nets on Mosquito Behaviour.} Washed and unwashed LLIN were evaluated in experimental huts for their effects on free-flying, wild $C x$. quinquefasciatus mosquitoes and for their ability to deter entry, repel, or drive mosquitoes out of houses, induce mortality, and inhibit blood-feeding. An untreated net was used as a negative control. A PermaNet $2.0\left(55 \mathrm{mg} / \mathrm{m}^{2}\right.$ deltamethrin) was used as a reference net. A net conventionally treated with deltamethrin and washed just before exhaustion was also used as another reference net. The point of exhaustion was determined by washing the conventionally treated net following Phase II 
protocol. After each washing, bioassays using WHO cones were performed. The last wash for which the net still causes $>80 \%$ mortality or $>95 \% \mathrm{KD}$ was considered to be the number of washes required before exhaustion. Finally, a PermaNet 2.0 washed 20 times was tested for comparison with PermaNet 3.0. Treatment arms were as follows:

(1) Untreated net (same fabric-polyester on the side/ polyethylene on top-and same design as of PermaNet 3.0, that is, with a border in the first $70 \mathrm{~cm}$ );

(2) PermaNet 2.0 washed 20 times;

(3) PermaNet 3.0 washed 20 times;

(4) PermaNet 2.0. unwashed;

(5) PermaNet 3.0 unwashed;

(6) Polyester net conventionally treated by deltamethrin at $25 \mathrm{mg} / \mathrm{m}^{2}$ and washed just before exhaustion $(<80 \%$ mortality in cone bioassay or $<95 \%$ knockdown after $1 \mathrm{~h})$.

2.4.1. Chemical Assay. An additional net per treatment arm was not actually placed in huts but kept for chemical assays. Pieces of nettings $\left(4 \times 50 \mathrm{~cm}^{2}\right)$ were taken from each of the five locations of these six nets according to WHO specifications before any washing to be subjected to chemical analysis. After washes were completed, pieces again were taken from each of the five locations of these six nets (including the unwashed nets) and shipped for chemical analysis. At the end of the experimental hut trial, new pieces were taken from each of the five locations of the six nets tested in the field (one per treatment arm). All samples for chemical analysis were sent to Vestergaard Frandsen SA after the field trial.

2.5. Larval and Pupal Mosquito Collections. Immature stages of mosquitoes (larvae and pupae) were prospected in Akodésséwa (N06 09'23.6"; E001 16 02.4") for Anopheles species and (N06 $09^{\prime} 42.3^{\prime \prime}$; E001 $\left.15^{\prime} 33.0^{\prime \prime}\right)$ for Culex species. Samples were transported to the laboratory (Laboratoire d'Entomologie Appliquée-LEA, University of Lomé) and reared under standard conditions in the insectarium $(12 \mathrm{Hr}$ : $12 \mathrm{Hr} \mathrm{LD} ; 72 \pm 2 \% \mathrm{RH})$. Emerged adults were fed on honey solution and allowed for a minimum of three days after emergence before used for susceptibility and efficacy test.

\subsection{Susceptibility Test with Insecticide Impregnated Papers.} Three-day-old unfed females (but on honey solution) of Anopheles gambiae and Culex were exposed in replicates of four to impregnated papers with the following insecticides: permethrin $(1 \%)$, deltamethrin $(0.05 \%, 0.5 \%)$, carbosulfan $(0.4 \%)$, DDT $(4 \%)$, bendiocarb $(0.1 \%)$, and chlorpyrifos methyl $(0.4 \%)$. Knockdown (KD) was recorded at time intervals for $1 \mathrm{hr}$ and mortality checked $24 \mathrm{hr}$ after exposure. Test with untreated paper was performed alongside each test to serve as controls and mortality was corrected ( $\geq 5 \%$ mortality) with Abbott's formula when necessary.
2.7. Efficacy Test on Mosquito Nets with Adult Mosquitoes. Three-day-old postemerged female Culex quinquefasciatus from Akodésséwa were subjected to three minutes contact exposure to all sides of each net: PermaNet 3.0 (unwashed and washed 20 times) and PermaNet 2.0 (unwashed and washed 20 times), using WHO cones. After exposure, mosquitoes were aspirated back into paper cups and observed for knockdown (KD) at time intervals for one hour and mortality observed after 24 hours after exposure. Tests were in replicates of five cones per net for the one-technology net (PermaNet 2.0) and five cones per side and per roof for the two-technology net (PermaNet 3.0). Mosquitoes were given honey solution and kept at $25^{\circ} \mathrm{C}, 70 \pm 2 \%$ relative humidity condition. Culex SLAB strains of mosquitoes were used as susceptible reference strains. Due to high resistance observed in the Culex mosquitoes after three minutes, a 30 minutes exposure contact time on nets was again tested.

2.8. Evaluation of Mosquito Nets in Experimental Huts. Six (6) experimental huts were constructed with concrete blocks, corrugated iron sheets for the roof, and lined with thick polyethylene sheeting. Huts were situated along a large water swarm area in the Akodésséwa district in Lomé, purposefully envisaged to breed mosquitoes. Each veranda-hut had the following measurements: $1.3 \mathrm{~mL}, 1.6 \mathrm{~mW}, 1.8-2 \mathrm{mH}$ (veranda), $1.7 \mathrm{~mL}, 3.2 \mathrm{~mW}, 2.1-2.3 \mathrm{mH}$ (hut), typical of the West Africa design hut type for phase II trials. The huts were spaced at intervals transversely. An exit window trap $(30 \mathrm{~cm} \times 60 \mathrm{~cm})$ is located on each side-wall part of the hut and two exit windows on the front-side wall (one on either side of door). A $1 \mathrm{~cm}$ (finger size) gap is left on each exit window for mosquito entry into huts.

The evaluations run between 11 February and 29 March 2008. The five treatments (including the untreated control net) were rotated through each of the six huts using a Latin square rotation design. A treatment was allocated to a hut for six consecutive nights before being rotated to another hut. A different net was used on each of the six nights of rotation. Sleepers were rotated between huts on successive nights in order to reduce the effect of variation in individual attractiveness to mosquitoes.

The primary outcomes measured in experimental huts were

(i) deterrence (reduction in hut entry relative to the control huts);

(ii) induced exophily (proportion of mosquitoes trying to exit early found in verandah-trap);

(iii) blood-feeding inhibition (reduction in blood feeding compared to control huts);

(iv) immediate and delayed mortality (proportion of dead mosquitoes).

2.9. Adult Mosquito Collection in Experimental Huts. Adult volunteers (sleepers) were consented and slept in the huts during the six-week-trial period (one complete Latin square rotation). Sleepers enter huts at dusk and remained till dawn. Mosquitoes were collected as per location and scored as dead 


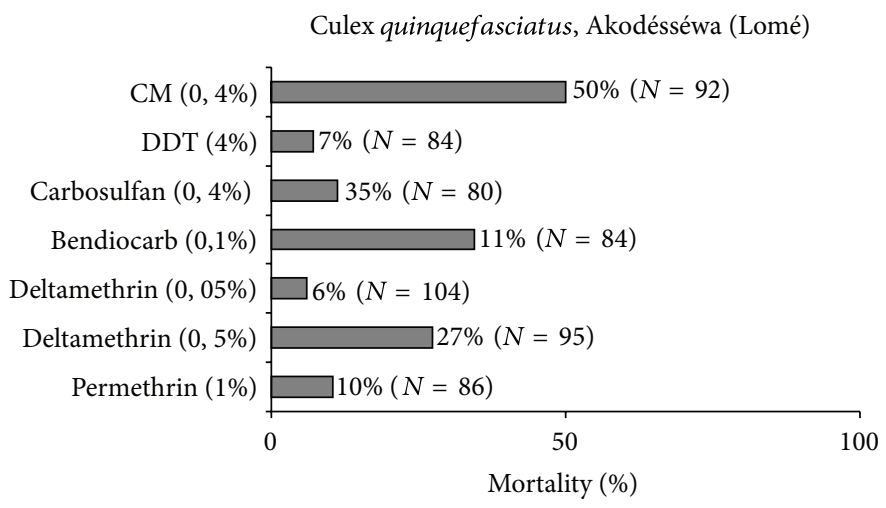

FIgUre 1: Insecticide susceptibility status of Culex quinquefasciatus at Akodésséwa, Togo (2008).

or alive in the laboratory after initial species identification. Live mosquitoes were subsequently put into paper cups, provided with honey solution, and delayed mortality observed 24 hrs after collection. Preliminary collection of mosquitoes was done in huts prior to start of trial to eliminate bias in attractiveness of sleepers to mosquitoes. In doing so, sleepers were rotated in $6 \times 6$ hut-night collection scheme to adjust for any bias in results interpretation.

2.10. Statistical Analysis. The number of mosquitoes of each species entering the huts, the proportion of mosquitoes that exited early, the proportion that was killed within the hut, and the proportion that successfully blood fed was compared by species and analysed using Mann Whitney rank test for numeric data and logistic regression for proportional data (e.g., XL-Stat software). Chi-square statistics were done using Fisher exact test. The clustering of observations made in one night, and controlling for any variation between huts and sleepers was controlled for. Comparisons between treatments were made by successively dropping treatments from the overall comparison, and this process allowed each treatment to be compared with every other one.

2.11. Ethical Considerations and Perceived Side Effects. Adult male participants were eligible for the trial. They were consented and objectives and procedures of the trial explained to them both in English and French. They were given an information consent sheet in English and in French to be taken away for further or future explanations of trial objectives and procedures to them by an independent person. They were then finally consented and made to sign a consent form before commencement of the trial. They were provided malaria prophylaxis during the entire period of the trial and two weeks after the completion of the trial. The trial received ethical clearance (endorsement) from the Ministry of Health of Togo and the University of Togo (Faculty of Science). Sleepers in huts were questioned at the end of the experiment about perceived adverse or beneficial side effects of the nets they had slept under.

\section{Results}

Susceptibility of Culex quinquefasciatus to tested insecticides is low (Figure 1). This ranged from $6 \%$ for deltamethrin to
$50 \%$ for the organophosphate chlorpyrifos methyl. The picture did not differ significantly when PBO was applied for $1 \mathrm{hr}$ contact before each insecticide. Mortalities increased for all insecticides except for the organophosphate CM (a decrease from $50 \%$ mortality to $37 \%$ in tested insect populations).

Based on trial endpoints (Table 5), PermaNet 3.0 in the unwashed state deterred $16.84 \%$ of Culex mosquitoes. After 20 washes, the net deterred $5.79 \%$ of mosquitoes from entering huts which is slightly lower than deterrence (6.84\%) offered by the unwashed PermaNet 2.0. PermaNet 3.0 was able to inhibit blood feeding by $70.97 \%$ and induce mosquitoes to exit huts by $50.48 \%$ at zero wash. After 20 washes, the net performed equally well by inducing $42.91 \%$ mosquitoes to exit huts and inhibited $67.06 \%$ mosquitoes from blood feeding. The polyester net conventionally treated and washed just before exhaustion did not have any significant effect on blood feeding inhibition nor mortality. Exophily was significantly induced by all treatments, except the net washed to exhaustion. Induced exophily was particularly significant with PermaNet 3.0 (64\% and 69\% for the washed and unwashed nets, resp.). Similarly, an important decrease of blood feeding was observed for PermaNet 3.0 (67\% and $71 \%$ for the washed and unwashed, resp.). To a lesser extent, PermaNet 2.0 inhibited 38\% and 46\% (washed and unwashed, resp.) of mosquitoes from successful blood feeding. Overall efficacy was good for Perma Net 3.0 after 20 washes (69\%) and when not washed (76\%).

\section{Discussion}

4.1. Susceptibility Tests. The high level of resistance to the main classes of insecticides (Table 1) confirms the multiresistance status of $C x$. quinquefasciatus worldwide and particularly in Africa [28] and therefore calls for better and comprehensive investigation of its resistance mechanisms locally. Some of the females have been kept by CREC to perform PCR diagnostic tests and biochemical assays for a better knowledge of resistant mechanisms.

4.2. Bioefficacy. Before any washing (Table 2), the fact that all treated nets are fully effective against the susceptible reference strain of $A n$. gambiae is an indication that deltamethrin is fully bioavailable regardless of the treatment, even when 
TABLE 1: Test for establishing point of exhaustion threshold.

\begin{tabular}{lcccc}
\hline $\begin{array}{l}\text { Number of } \\
\text { washes }\end{array}$ & $\begin{array}{c}\% \mathrm{KD} \\
60 \mathrm{~min}\end{array}$ & $\begin{array}{c}\text { \% Mort. } \\
24 \mathrm{hrs}\end{array}$ & $N$ & $\begin{array}{c}\text { \% Mort. } \\
\text { control }(n)\end{array}$ \\
\hline 0 wash & $100^{\mathrm{a}}$ & $100^{\mathrm{a}}$ & 60 & $0.00(56)$ \\
1 wash & $100^{\mathrm{a}}$ & $100^{\mathrm{a}}$ & 55 & $0.00(56)$ \\
2 washes & $100^{\mathrm{a}}$ & $100^{\mathrm{a}}$ & 59 & $0.00(56)$ \\
3 washes & $98.31^{\mathrm{a}}$ & $86.44^{\mathrm{b}}$ & 59 & $0.00(56)$ \\
4 washes & $91.80^{\mathrm{a}}$ & $65.57^{\mathrm{c}}$ & 61 & $0.00(56)$ \\
\hline
\end{tabular}

$N$ and (n) indicate numbers tested in test group and in control, respectively, KD: knockdown, Mort: mortality.

${ }^{\mathrm{a}, \mathrm{b}, \mathrm{c}}$ Values (or data) in the same row sharing a letter superscript do not differ significantly $(P>0.05)$.

TABLE 2: Initial bioefficacy of the treated nets.

\begin{tabular}{lccc}
\hline Treatment & \% KD & \% Mort. & $n$ \\
& $60 \mathrm{~min}$ & $24 \mathrm{hrs}$ & \\
\hline Untreated polyester net & $0.00^{\mathrm{a}}$ & $1.89^{\mathrm{a}}$ & 53 \\
PermaNet 2.0 unwashed & $100^{\mathrm{b}}$ & $100^{\mathrm{b}}$ & 61 \\
PermaNet 3.0 unwashed & $100^{\mathrm{b}}$ & $100^{\mathrm{b}}$ & 60 \\
PermaNet 3.0 unwashed-roof (+ PBO) & $100^{\mathrm{b}}$ & $100^{\mathrm{b}}$ & 54 \\
PermaNet 2.0 to be washed 20 times & $100^{\mathrm{b}}$ & $100^{\mathrm{b}}$ & 49 \\
PermaNet 3.0 to be washed 20 times & $100^{\mathrm{b}}$ & $100^{\mathrm{b}}$ & 52 \\
$\begin{array}{l}\text { PermaNet 3.0 washed 20 times—roof } \\
\text { (+ PBO) }\end{array}$ & $100^{\mathrm{b}}$ & $100^{\mathrm{b}}$ & 51 \\
$\begin{array}{l}\text { Polyester net to be washed just before } \\
\text { exhaustion }\end{array}$ & $100^{\mathrm{b}}$ & $100^{\mathrm{b}}$ & 60 \\
\hline
\end{tabular}

${ }^{a}$ Values (or data) in the same column sharing a letter superscript do not differ significantly $(P>0.05)$.

using the long lasting technology. The 3 washes required just before exhaustion (Table 1) are a little bit higher than the 2 washes usually observed with other Phase II evaluations (Dawa net, K-O Tables 1, 2, and 3 and NetProtect). This slight difference can be considered as an acceptable "biological variability" between bioassays from one test to another. Resistance to washing before or after field testing (Tables 3 and 4) is remarkably important on both PermaNet 3.0 (including the one with roof treated with $\mathrm{PBO}$ ) and PermaNet 2.0 (despite the fact that a slight but significant decrease in mortality had been observed on PermaNet 2.0 washed 20 times even before field testing).

4.3. Field Efficacy. Results obtained with Cx. quinquefasciatus in the control arm (untreated) do not differ radically from those obtained with An. gambiae in other experimental hut studies. Natural exophily is comparable (30-40\%) as well as natural mortality (5-10\%). Blood feeding is, however, less important with this species (48\%) compared to the rates obtained with An. gambiae (i.e., 76\% in September in Kou Valley, Burkina Faso). Additional studies against Culex species are needed to know whether this difference in blood feeding is interspecific (difference in vector behaviour) and not due to intertrial variability.

In a nutshell, $C x$. quinquefasciatus is less affected by PermaNet 3.0 than An. gambiae: deterrence is practically
TABLE 3: Resistance to washing before field testing. After all washes were completed (February 10, 2008), nets were tested before being installed in experimental huts (Table 3). All treated nets were fully effective in terms of knockdown effect and mortality except PermaNet 2.0 washed 20 times $(P=0.013)$. Knockdown and mortality were recorded at 10 minutes interval using WHO cones, after washing and before field testing, after $3 \mathrm{~min}$ exposure of susceptible reference strain of An. gambiae (Kisumu strain).

\begin{tabular}{lccc}
\hline Treatment & \% KD & \% Mort. & $n$ \\
& 60 min & 24 hrs & $n$ \\
\hline Untreated polyester net & $0.00^{\mathrm{a}}$ & $0.00^{\mathrm{a}}$ & 59 \\
PermaNet 2.0 unwashed & $100^{\mathrm{b}}$ & $100^{\mathrm{b}}$ & 56 \\
PermaNet 3.0 unwashed & $100^{\mathrm{b}}$ & $100^{\mathrm{b}}$ & 65 \\
PermaNet 3.0 unwashed-roof (+ PBO) & $100^{\mathrm{b}}$ & $100^{\mathrm{b}}$ & 63 \\
PermaNet 2.0 washed 20 times & $93.65^{\mathrm{b}}$ & $84.13^{\mathrm{c}}$ & 63 \\
PermaNet 3.0 washed 20 times & $100^{\mathrm{b}}$ & $100^{\mathrm{b}}$ & 69 \\
$\begin{array}{l}\text { PermaNet 3.0 washed 20 times-roof } \\
\text { (+ PBO) }\end{array}$ & $100^{\mathrm{b}}$ & $100^{\mathrm{b}}$ & 65 \\
$\begin{array}{l}\text { Polyester net washed just before } \\
\text { exhaustion }\end{array}$ & $98.33^{\mathrm{b}}$ & $93.33^{\mathrm{b}}$ & 60 \\
\hline
\end{tabular}

${ }^{a}$ Values (or data) in the same column sharing a letter superscript do not differ significantly $(P>0.05)$.

TABLE 4: Resistance to washing after field testing. After the end of the field trial (March 29, 2008), nets collected from huts were tested. Results yielded knockdown and mortality rates almost similar to those recorded immediately after washing and before testing. Knockdown and mortality were recorded at 10 minutes interval using WHO cones, after washing and after field testing, after 3 min exposure of susceptible reference strain of An. gambiae (Kisumu strain).

\begin{tabular}{lccc}
\hline Treatment & \% KD & \% Mort. & $n$ \\
& 60 min & 24 hrs & $n$ \\
\hline Untreated polyester net & $0.00^{\mathrm{a}}$ & $0.00^{\mathrm{a}}$ & 59 \\
PermaNet 2.0 unwashed & $100^{\mathrm{b}}$ & $100^{\mathrm{b}}$ & 57 \\
PermaNet 3.0 unwashed & $100^{\mathrm{b}}$ & $100^{\mathrm{b}}$ & 57 \\
PermaNet 3.0 unwashed-roof (+ PBO) & $100^{\mathrm{b}}$ & $100^{\mathrm{b}}$ & 58 \\
PermaNet 2.0 washed 20 times & $94.23^{\mathrm{b}}$ & $94.23^{\mathrm{b}}$ & 52 \\
PermaNet 3.0 washed 20 times & $100^{\mathrm{b}}$ & $100^{\mathrm{b}}$ & 58 \\
$\begin{array}{l}\text { PermaNet 3.0 washed 20 times-roof } \\
\text { (+ PBO) }\end{array}$ & $100^{\mathrm{b}}$ & $100^{\mathrm{b}}$ & 54 \\
Polyester net washed just before & $89.66^{\mathrm{c}}$ & $94.83^{\mathrm{b}}$ & 58 \\
exhaustion & & & \\
\hline
\end{tabular}

${ }^{a}$ Values (or data) in the same column sharing a letter superscript do not differ significantly $(P>0.05)$.

zero, mortality is very low (only $7 \%$ overall insecticidal effect with PermaNet 3.0 unwashed), and induced exophily is moderate. However, blood feeding inhibition remains as elevated as for pyrethroid resistant An. gambiae in Burkina Faso, leading to maintain a good personal protection despite the high level of pyrethroid resistance of local populations of $C x$. quinquefasciatus. Moreover, this personal protection is much higher with PermaNet 3.0, washed and unwashed (69 and 76\%, resp.) than with PermaNet 2.0 (49 and 22\%, resp.). 
TABLE 5: Summary of results for free-flying wild Culex quinquefasciatus (36 nights) in experimental huts (Akodésséwa, Togo).

\begin{tabular}{|c|c|c|c|c|c|c|}
\hline & $\begin{array}{c}\text { Arm } 1 \\
\text { Untreated } \\
\text { net } \\
\end{array}$ & $\begin{array}{c}\text { Arm } 2 \\
\text { PermaNet } 2.0 \\
20 \text { washes }\end{array}$ & $\begin{array}{c}\text { Arm } 3 \\
\text { PermaNet } 3.0 \\
20 \text { washes } \\
\end{array}$ & $\begin{array}{c}\text { Arm } 4 \\
\text { PermaNet } 2.0 \\
\text { unwashed } \\
\end{array}$ & $\begin{array}{c}\text { Arm } 5 \\
\text { PermaNet } 3.0 \\
\text { unwashed } \\
\end{array}$ & $\begin{array}{c}\text { Arm } 6 \\
\text { Polyester net } \\
\text { before exhaust. }\end{array}$ \\
\hline Total females caught & 190 & 241 & 179 & 177 & 158 & 278 \\
\hline Females caught/night & $5.28^{\mathrm{a}}$ & $6.69^{\mathrm{b}}$ & $4.97^{\mathrm{a}}$ & $4.92^{\mathrm{a}}$ & $4.39^{\mathrm{a}}$ & $7.72^{\mathrm{b}}$ \\
\hline Deterrence (\%) & - & - & 5.79 & 6.84 & 16.84 & - \\
\hline Total females verandah & 71 & 124 & 115 & 100 & 109 & 122 \\
\hline Exophily (\%) & $37.37^{\mathrm{a}}$ & $51.45^{\mathrm{b}}$ & $64.25^{\mathrm{c}, \mathrm{d}}$ & $56.50^{\mathrm{b}, \mathrm{c}}$ & $68.99^{\mathrm{d}}$ & $43.88^{\mathrm{a}}$ \\
\hline 95\% Confidence limits & $30.84-44.53$ & $45.15-57.70$ & $56.88-70.85$ & $49.07-63.58$ & $61.24-75.60$ & $38.18-49.80$ \\
\hline Induced Exophily (\%) & - & 22.49 & 42.91 & 30.54 & 50.48 & NS \\
\hline Total females blood fed & 87 & 68 & 27 & 44 & 21 & 108 \\
\hline Blood fed (\%) & $45.79^{\mathrm{a}}$ & $28.22^{\mathrm{b}}$ & $15.08^{\mathrm{c}}$ & $24.86^{\mathrm{b}}$ & $13.29^{\mathrm{c}}$ & $38.85^{\mathrm{a}}$ \\
\hline 95\% Confidence limits & $38.86-52.93$ & $22.98-34.32$ & $10.72-21.32$ & $19.17-31,90$ & $9.02-19.79$ & $33.33-44.75$ \\
\hline Blood feeding inhibition (\%) & - & 38.38 & 67.06 & 45.71 & 70.97 & NS \\
\hline Personal protection & & 21.84 & 68.97 & 49.43 & 75.86 & 0.00 \\
\hline Total females dead & 20 & 30 & 31 & 23 & 32 & 27 \\
\hline Overall mortality (\%) & $10.53^{\mathrm{a}, \mathrm{b}}$ & $12.45^{\mathrm{a}, \mathrm{b}}$ & $17.32^{\mathrm{b}}$ & $12.99^{\mathrm{a}, \mathrm{b}}$ & $20.25^{\mathrm{b}}$ & $9.71^{\mathrm{a}}$ \\
\hline 95\% Confidence limits & $7.06-15.99$ & $8.97-17.42$ & $12.61-23.78$ & $8.96-19.03$ & $14.86-27.44$ & $6.86-13.96$ \\
\hline Corrected for control (\%) & - & 2.15 & 7.59 & 2.76 & 10.87 & - \\
\hline Overall insecticidal effect & & 5.88 & 6.47 & 1.76 & 7.06 & 4.12 \\
\hline
\end{tabular}

${ }^{a}$ Values (or data) in the same row sharing a letter superscript do not differ significantly $(P>0.05)$.

The fact that mortality is low and blood feeding inhibition is high is probably the result of the behavioural response of Cx. quinquefasciatus to pyrethroids: contact time with the treated fabrics is not long enough to kill mosquitoes but enough to prevent blood feeding. The efficacy of the new mosaic net is better explained by the overall personal protection it offers to an individual. Comparatively, it performed better in protection of sleepers from mosquito bites which was nearly two times that of the conventionally used net. This protective effect is higher than the conventional net, after the recommended 20 minimum washes. After trials, contact bioassays revealed that all nets were effective in killing susceptible Anopheles gambiae (kisumu strain) except the manually treated net just washed to exhaustion. This confirms the rapid loss of efficacy of nets manually treated. Also, these results support multicountry trials [11] that demonstrated the ability of retention of insecticide properties by long lasting insecticide nets even after 20 minimum washes.

\section{Conclusions}

Resistance in Culex quinquefasciatus is high in Lomé, particularly in Akodésséwa. Further toxicology studies of water samples from breeding sites of this species are recommended to give concrete and better understanding of the high resistance observed in this study. Innovative vector control measures are seriously encouraged and constant research on vector species dynamics is welcomed, as it demonstrates at any given time vector resistance and susceptibility status.
The fact that all treated nets (before any washing) were fully effective against the susceptible reference strain of $A n$. gambiae is an indication that deltamethrin is fully bioavailable regardless of the treatment, even when using the long lasting technology. The new technology incorporated in the mosaic net evaluated is good. However, $C x$. quinquefasciatus is less affected by PermaNet 3.0. Deterrence is practically negligible and mortality very low. However, blood feeding inhibition remains high which resultantly gives a good personal protection despite the high level of pyrethroid resistance of local populations of Cx. quinquefasciatus. Moreover, this personal protection is much higher with PermaNet 3.0 , washed and unwashed (69\% and $76 \%$, resp.) than with PermaNet 2.0 (22\% and 49\%) for washed and unwashed, respectively.

Results for PermaNet 3.0 are rather encouraging. PermaNet 3.0 succeeded in reducing blood feeding and thus nuisance. It is well known that in order for LLINS to be used adequately against malaria vectors, they also need to be effective against nuisance pest mosquitoes, particularly $C x$. quinquefasciatus in tropical urban environment, as they will be widely accepted by the local populations.

\section{Conflict of Interests}

The manufacturer of PermaNet 3.0 (Vestergaard Frandsen), played no role in the design or implementation of the research, decision to publish or drafting the paper. All authors declare that they have no conflict of interests. 


\section{Authors' Contribution}

J.-M. Hougard is the principal investigator. D. B. Dery implemented the research as part of his M.S. thesis dissertation and drafted the paper. J. Chabi and G. Apetogbo contributed in field data collection. G. K. Ketoh and I. A. Glitho coordinated data collection in Lomé. T. Baldet gave technical inputs in the implementation of the project and data analysis. All authors read and approved the paper.

\section{Acknowledgments}

Individuals, groups, and institutions contributed to the research. Sincere gratitude goes to sleepers for consenting to be part of the research, Ministry of Health of Togo, University of Lomé (Faculty of Science), Laboratoire de Entomologie Appliquée (LEA) staff and students, ABC network, CREC, MIE technical and steering committee, and University of Abomey-Calavi (Benin).

\section{References}

[1] J. M. Hougard, T. Martin, P. F. Guillet et al., "Preliminary field testing of a long-lasting insecticide-treated hammock against Anopheles gambiae and Mansonia spp. (Diptera: Culicidae) in West Africa," Journal of Medical Entomology, vol. 44, no. 4, pp. 651-655, 2007.

[2] D. H. Molyneux, G. Barnish, S. Looreesuwan, B. Liese, and J. Hemingway, "Global campaign to eradicate malaria. Malaria is paradigm of an emergent disease," BMJ, vol. 323, no. 7312, p. $571,2001$.

[3] F. Binka and P. Akweongo, "Prevention of malaria using ITNs: potential for achieving the millennium development goals," Current Molecular Medicine, vol. 6, no. 2, pp. 261-267, 2006.

[4] B. Ostyn, V. Vanlerberghe, A. Picado et al., "Vector control by insecticide-treated nets in the fight against visceral leishmaniasis in the Indian subcontinent, what is the evidence?" Tropical Medicine and International Health, vol. 13, no. 8, pp. 1073-1085, 2008.

[5] S. K. Sharma, A. K. Upadhyay, M. A. Haque et al., "Village-scale evaluation of mosquito nets treated with a tablet formulation of deltamethrin against malaria vectors," Medical and Veterinary Entomology, vol. 19, no. 3, pp. 286-292, 2005.

[6] F. N. Binka, A. Kubaje, M. Adjuik et al., "Impact of permethrin impregnated bednets on child mortality in KassenaNankana district, Ghana: a randomized controlled trial," Tropical Medicine and International Health, vol. 1, no. 2, pp. 147-154, 1996.

[7] O. Müller, K. Ido, and C. Traoré, "Evaluation of a prototype long-lasting insecticide-Treated mosquito net under field conditions in rural Burkina Faso," Transactions of the Royal Society of Tropical Medicine and Hygiene, vol. 96, no. 5, pp. 483-484, 2002.

[8] R. N'Guessan, F. Darriet, J. M. C. Doannio, F. Chandre, and P. Carnevale, "Olyset Net efficacy against pyrethroid-resistant Anopheles gambiae and Culex quinquefasciatus after 3 years' field use in Côte d'Ivoire," Medical and Veterinary Entomology, vol. 15, no. 1, pp. 97-104, 2001.

[9] M. A. Kulkarni, R. Malima, F. W. Mosha et al., "Efficacy of pyrethroid-treated nets against malaria vectors and nuisance-biting mosquitoes in Tanzania in areas with long-term insecticide-treated net use," Tropical Medicine and International Health, vol. 12, no. 9, pp. 1061-1073, 2007.

[10] R. N’Guessan, P. Boko, A. Odjo, B. Knols, M. Akogbeto, and M. Rowland, "Control of pyrethroid-resistant Anopheles gambiae and Culex quinquefasciatus mosquitoes with chlorfenapyr in Benin," Tropical Medicine and International Health, vol. 14, no. 4, pp. 389-395, 2009.

[11] K. Graham, M. H. Kayedi, C. Maxwell et al., "Multicountry field trials comparing wash-resistance of PermaNet and conventional insecticide-treated nets against anopheline and culicine mosquitoes," Medical and Veterinary Entomology, vol. 19, no. 1, pp. 72-83, 2005.

[12] S. Sathantriphop, C. Ketavan, A. Prabaripai et al., "Susceptibility and avoidance behavior by Culex quinquefasciatus say to three classes of residual insecticides," Journal of Vector Ecology, vol. 31, no. 2, pp. 266-274, 2006.

[13] S. Sathantriphop, P. Paeporn, and K. Supaphathom, "Detection of insecticides resistance status in Culex quinquefasciatus and Aedes aegypti to four major groups of insecticides," Tropical biomedicine, vol. 23, no. 1, pp. 97-101, 2006.

[14] A. N. Asidi, R. N'Guessan, R. A. Hutchinson, M. TraoreLamizana, P. Carnevale, and C. F. Curtis, "Experimental hut comparisons of nets treated with carbamate or pyrethroid insecticides, washed or unwashed, against pyrethroid-resistant mosquitoes," Medical and Veterinary Entomology, vol. 18, pp. 134-140, 2004.

[15] S. H. P. P. Karunaratne, J. Hemingway, J. Hemingway, K. G. I. Jayawardena, V. Dassanayaka, and A. Vaughan, "Kinetic and molecular differences in the amplified and non-amplified esterases from insecticide-resistant and susceptible Culex quinquefasciatus mosquitoes," Journal of Biological Chemistry, vol. 270, no. 52, pp. 31124-31128, 1995.

[16] J. Hemingway, "Insecticide resistance in malaria vectors: a new approach to an old subject," Parassitologia, vol. 41, no. 1-3, pp. 315-318, 1999.

[17] G. Gentile, F. Santolamazza, C. Fanello, V. Petrarca, A. Caccone, and A. Della Torre, "Variation in an intron sequence of the voltage-gated sodium channel gene correlates with genetic differentiation between Anopheles gambiae s.s. molecular forms," Insect Molecular Biology, vol. 13, no. 4, pp. 371-377, 2004.

[18] H. Ranson, L. Rossiter, F. Ortelli et al., "Identification of a novel class of insect glutathione S-transferases involved in resistance to DDT in the malaria vector Anopheles gambiae," Biochemical Journal, vol. 359, no. 2, pp. 295-304, 2001.

[19] J. M. Hougard, S. Duchon, F. Darriet, M. Zaim, C. Rogier, and P. Guillet, "Comparative performances, under laboratory conditions, of seven pyrethroid insecticides used for impregnation of mosquito nets," Bulletin of the World Health Organization, vol. 81, no. 5, pp. 324-333, 2003.

[20] C. Pennetier, V. Corbel, P. Boko et al., "Synergy between repellents and non-pyrethroid insecticides strongly extends the efficacy of treated nets against Anopheles gambiae," Malaria Journal, vol. 6, article 38, 2007.

[21] C. F. Curtis, J. Myamba, and T. J. Wilkes, "Comparison of different insecticides and fabrics for anti-mosquito bednets and curtains," Medical and Veterinary Entomology, vol. 10, no. 1, pp. 1-11, 1996.

[22] J. M. Hougard, V. Corbel, R. N'Guessan et al., "Efficacy of mosquito nets treated with insecticide mixtures or mosaics against insecticide resistant Anopheles gambiae and Culex quinquefasciatus (Diptera: Culicidae) in Côte d'Ivoire," Bulletin of Entomological Research, vol. 93, no. 6, pp. 491-498, 2003. 
[23] H. Ranson, M. G. Paton, B. Jensen et al., "Genetic mapping of genes conferring permethrin resistance in the malaria vector, Anopheles gambiae," Insect Molecular Biology, vol. 13, no. 4, pp. 379-386, 2004.

[24] F. Chandre and J. M. Hougard, "Ivermectin as a systemic insecticide on Culex quinquefasciatus and Simulium squamosum," Bulletin de la Societe de Pathologie Exotique, vol. 92, no. 1, pp. 71-72, 1999.

[25] V. Corbel, R. N’Guessan, C. Brengues et al., "Multiple insecticide resistance mechanisms in Anopheles gambiae and Culex quinquefasciatus from Benin, West Africa," Acta Tropica, vol. 101, no. 3, pp. 207-216, 2007.

[26] S. C. Britch, K. J. Linthicum, W. W. Wynn et al., "Longevity and efficacy of bifenthrin treatment on desert-pattern US military camouflage netting against mosquitoes in a hot-arid environment," Journal of the American Mosquito Control Association, vol. 27, no. 3, pp. 272-279, 2011.

[27] V. Corbel, J. Chabi, R. D. Dabire et al., "Field efficacy of a new mosaic long-lasting mosquito net (PermaNet 3.0) against pyrethroid-resistant malaria vectors: a multi centre study in Western and Central Africa," Malaria Journal, vol. 9, article 113, 2010.

[28] R. C. Malima, R. M. Oxborough, P. K. Tungu et al., "Behavioural and insecticidal effects of organophosphate-, carbamate- and pyrethroid-treated mosquito nets against African malaria vectors," Medical and Veterinary Entomology, vol. 23, no. 4, pp. 317-325, 2009. 


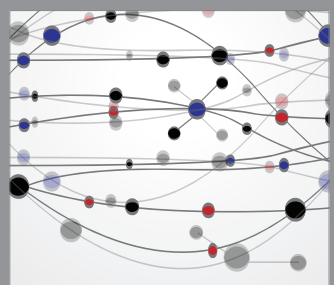

The Scientific World Journal
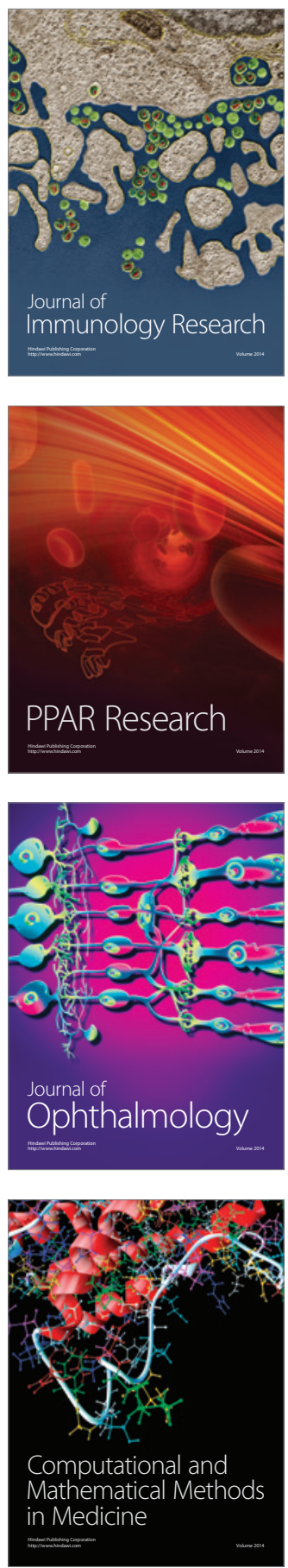

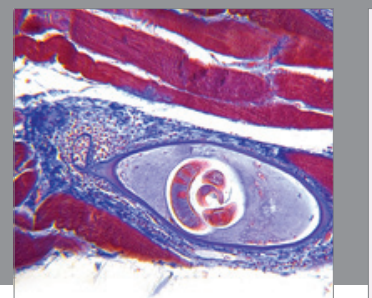

Gastroenterology

Research and Practice
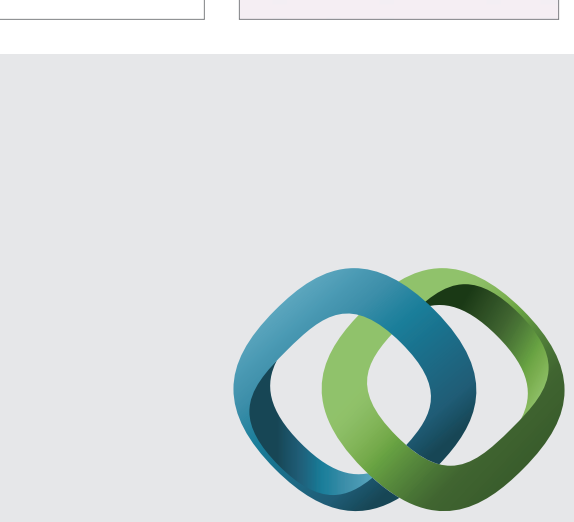

\section{Hindawi}

Submit your manuscripts at

http://www.hindawi.com
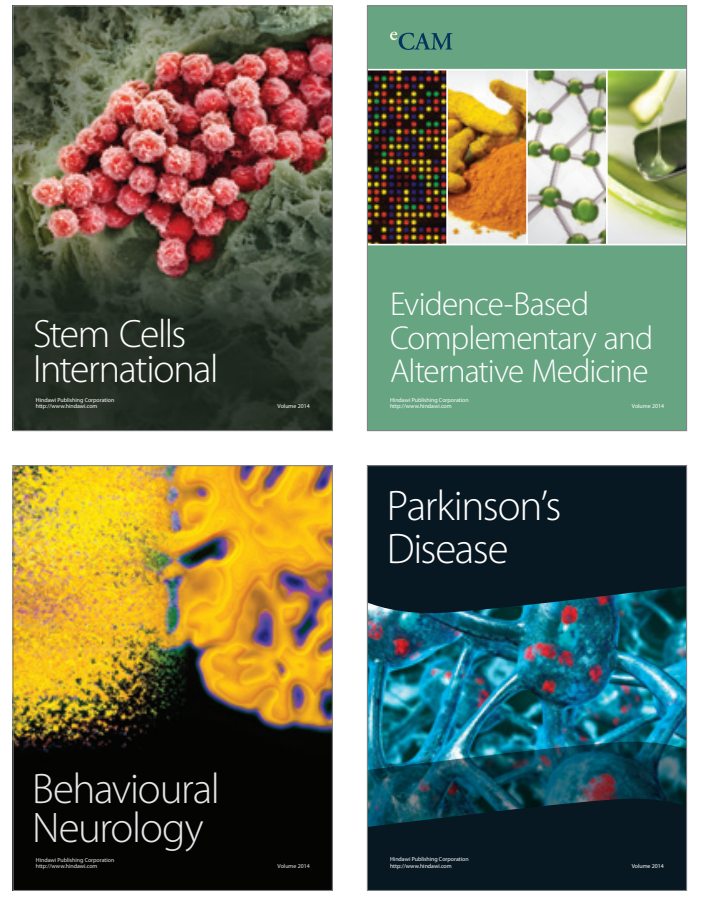
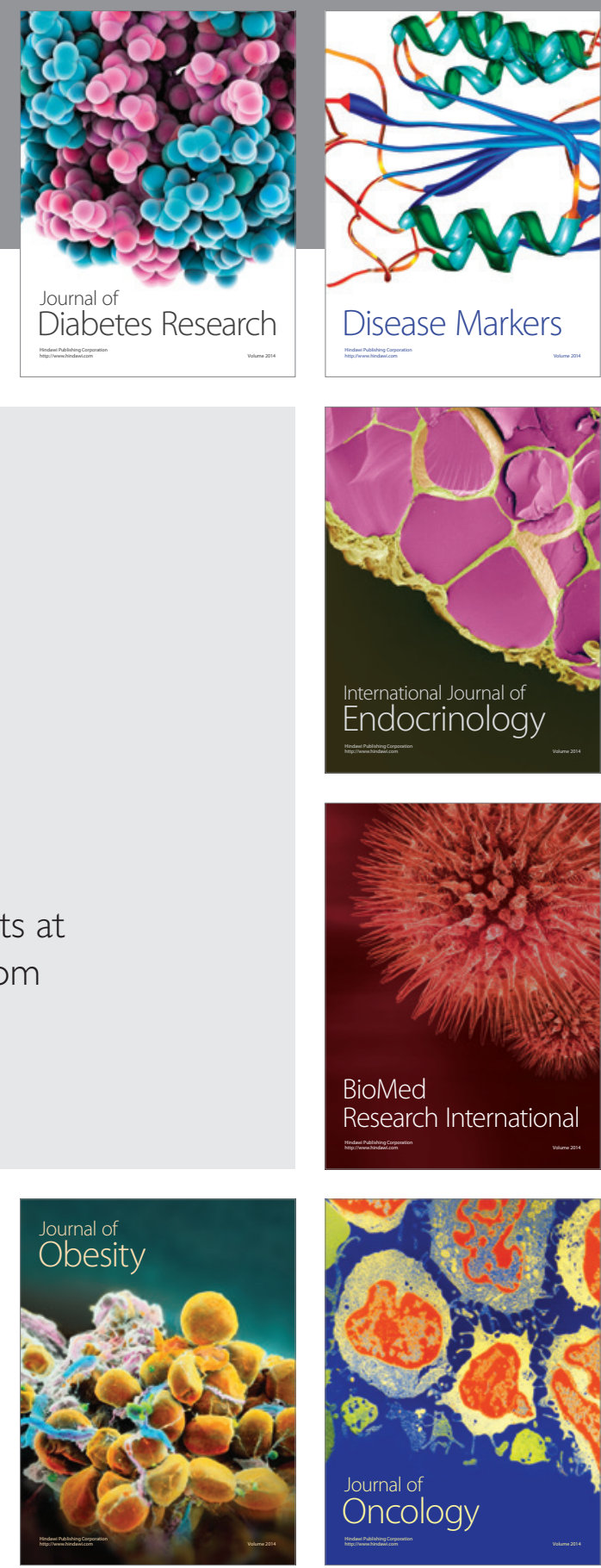

Disease Markers
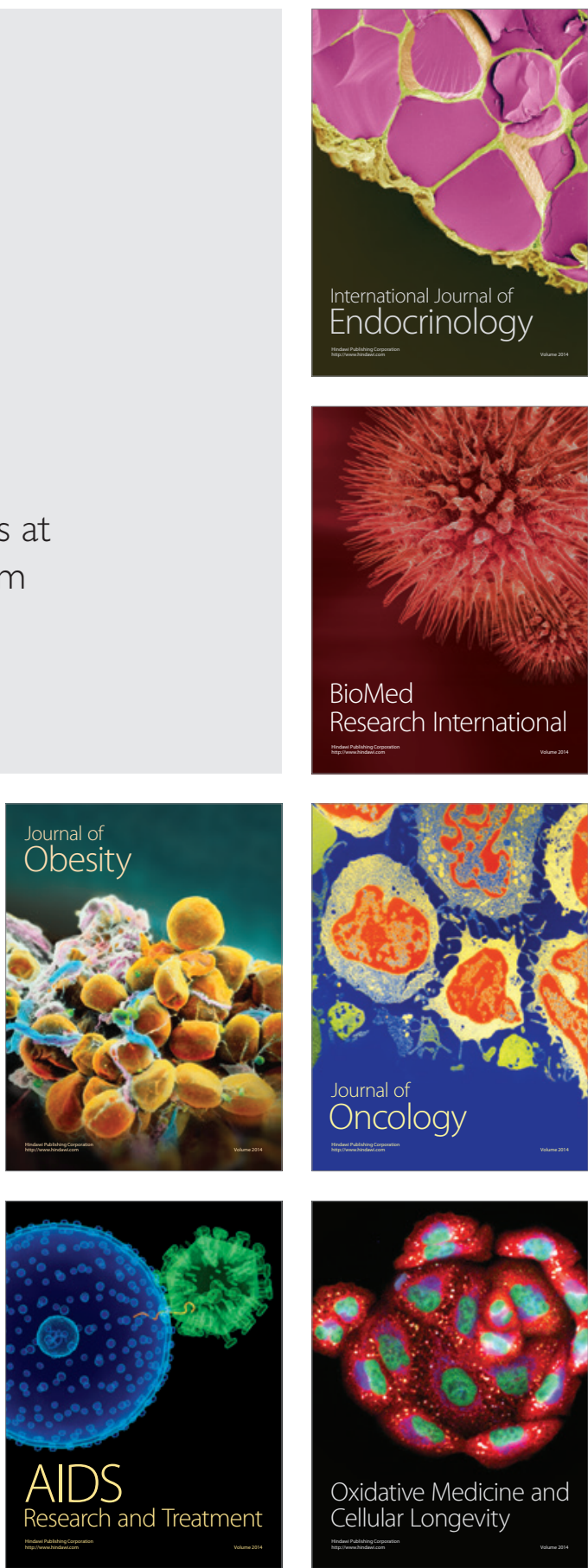